\title{
Neuronal Mechanisms and Attentional Modulation of Corticothalamic Alpha Oscillations
}

\author{
Anil Bollimunta, ${ }^{1}$ Jue Mo, ${ }^{1}$ Charles E. Schroeder, ${ }^{2,3}$ and Mingzhou Ding ${ }^{1}$ \\ ${ }^{1}$ J. Crayton Pruitt Family Department of Biomedical Engineering, University of Florida, Gainesville, Florida 32611, ${ }^{2}$ Nathan Kline Institute for Psychiatric \\ Research, Orangeburg, New York 10962, and ${ }^{3}$ Columbia University College of Physicians and Surgeons, New York, New York 10027
}

Field potential oscillations in the $\sim 10 \mathrm{~Hz}$ range are known as the alpha rhythm. The genesis and function of alpha has been the subject of intense investigation for the past 80 years. Whereas early work focused on the thalamus as the pacemaker of alpha rhythm, subsequent slice studies revealed that pyramidal neurons in the deep layers of sensory cortices are capable of oscillating in the alpha frequency range independently. How thalamic and cortical generating mechanisms in the intact brain might interact to shape the organization and function of alpha oscillations remains unclear. We addressed this problem by analyzing laminar profiles of local field potential and multiunit activity (MUA) recorded with linear array multielectrodes from the striate cortex of two macaque monkeys performing an intermodal selective attention task. Current source density (CSD) analysis was combined with CSD-MUA coherence to identify intracortical alpha current generators and assess their potential for pacemaking. Coherence and Granger causality analysis was applied to delineate the patterns of interaction among different alpha current generators. We found that (1) separable alpha current generators are located in superficial, granular, and deep layers, with both layer 4C and deep layers containing primary local pacemaking generators, suggesting the involvement of the thalamocortical network, and (2) visual attention reduces the magnitude of alpha oscillations as well as the level of alpha interactions, consistent with numerous reports of occipital alpha reduction with visual attention in human EEG. There is also indication that alpha oscillations in the lateral geniculate cohere with those in V1.

\section{Introduction}

Alpha rhythm $(\sim 10 \mathrm{~Hz})$ is a hallmark of spontaneous EEG oscillatory activity over the occipital-parietal cortex of awake humans (Shaw, 2003; Niedermeyer, 2005; Buzsaki, 2006). The physiological mechanism generating alpha is an open question. The prevailing notion before the 1970s was that the thalamus is the sole pacemaker of cortical alpha (Andersen and Andersson, 1968). In the 1970s, this notion was challenged by a series of in vivo studies (Lopes da Silva et al., 1973a,b, 1980; Lopes da Silva and Storm van Leeuwen, 1977), which showed that the alpha oscillations could be of a cortical origin, with large layer 5 pyramidal neurons acting as pacemakers (Steriade et al., 1990; Lopes da Silva, 1991). Support for this cortical alpha genesis hypothesis comes from in vitro slice preparations from the primary somatosensory cortex in the rat (Silva et al., 1991; Castro-Alamancos and Connors, 1996; Flint and Connors, 1996) as well as from laminar recordings in extrastriate cortex of awake-behaving macaques (Bollimunta et al., 2008).

It is a long-held notion that brain oscillations involve recurrent thalamocortical networks (Steriade et al., 1990). In vision, the topographic pattern of connections between the relay cells of

\footnotetext{
Received 0ct. 25, 2010; revised Jan. 28, 2011; accepted Feb. 4, 2011.

Author contributions: M.D. and C.E.S. designed research; A.B. and J.M. performed research; C.E.S. contributed unpublished reagents/analytic tools; A.B. and J.M. analyzed data; M.D., A.B., and C.E.S. wrote the paper.

This work was supported by National Institutes of Health Grants MH79388, MH60358, and MH87275. We thank A. D. Mehta and I. Ulbert for assistance with data collection.

Correspondence should be addressed to Mingzhou Ding at the above address. E-mail: mding@bme.ufl.edu. DOI:10.1523/JNEUROSCI.5580-10.2011

Copyright $\odot 2011$ the authors $\quad 0270-6474 / 11 / 314935-09 \$ 15.00 / 0$
}

the lateral geniculate nucleus (LGN) and the primary visual cortex (Jones, 2002) provide the anatomical substrate for such a network. Recent in vitro studies of thalamic nuclei in cats have found that a subset of the relay cells in LGN have the ability to oscillate at alpha frequencies through high-threshold bursting and suggested that these cells are a source of alpha activity in V1 (Hughes et al., 2004; Hughes and Crunelli, 2005; Lörincz et al., 2008). The first goal of the present study was to investigate the possible interplay between cortical and thalamic alphagenerating mechanisms in V1.

Human scalp EEG recordings have demonstrated that the alpha rhythm attenuates during tasks requiring externally deployed visual attention (Worden et al., 2000; Rajagovindan and Ding, 2010). In contrast, in a variety of experimental paradigms requiring internally directed attention, such as working memory and mental imagery, the alpha rhythm increases in magnitude with task demand (Jensen et al., 2002). The interpretation of these findings remains debated (Palva and Palva, 2007; Mo et al., 2011). The poor spatial resolution of scalp EEG is a limiting factor. The second goal of the present study was to investigate how attention modulates alpha activity at the level of local neuronal ensembles and their interactions in V1.

To accomplish these goals, we analyzed laminar profiles of local field potential (LFP) and multiunit activity (MUA) from the striate cortex V1 of two macaque monkeys performing an intermodal selective attention task (visual versus auditory). Similar to the recently published analysis of alpha activity in extrastriate cortex recorded from the same subjects (Bollimunta et al., 2008), current source density (CSD) analysis is combined with multi- 


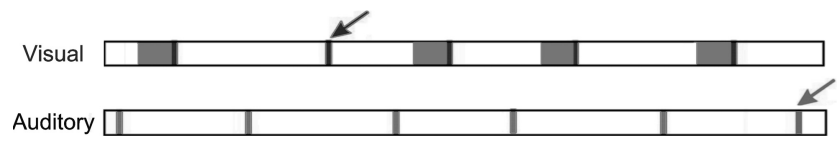

Figure 1. Time course of stimulus presentation in both the visual and auditory domain. The vertical bars represent stimuli, and deviant stimuli are indicated by arrows. The shaded interval before each standard visual stimulus defines the prestimulus time period of $200 \mathrm{~ms}$ in duration from which data were extracted for analysis in the attend-visual and ignore-visual conditions. For the auditory-only condition, no visual stimuli were presented.

variate spectral methods to identify laminar generators of alpha rhythm and to evaluate their patterns of interaction. The functional relevance of spectral power, coherence, and Granger causality was examined by comparing them across three different experimental conditions: auditory stimulation only, attendauditory during bimodal stimulation, and attend-visual during bimodal stimulation.

\section{Materials and Methods}

\section{Experiment}

All surgical, training, and other relevant aspects of the experimental procedure were approved by the Institutional Animal Care and Use Committee.

Paradigm. Two male macaque monkeys (Macaca fascicularis), B and V, were trained to perform an intermodal selective attention task (visual vs auditory) (Mehta et al., 2000a,b; Lakatos et al., 2008). Auditory stimulation consisted of a stream of pure tones ( $100 \mathrm{~ms}$ duration), jittered in onset time over a $\pm 200 \mathrm{~ms}$ range around a mean rate of $1.5 \mathrm{~Hz}$. Eighty-six percent of the tones were repetitive "standards" of a single frequency, and $14 \%$ were "deviants" created by a slight change in frequencies. Visual stimulation consisted of $10 \mu$ s flashes, subtending 20 retinal degrees surrounding fixation, in a stream that was jittered over the same range and around the same mean rate as the auditory stimuli. The standard and deviant flashes (slight change in intensity) had the same $86 \%$ versus $14 \%$ ratio. There are three experimental conditions. In condition 1 , the monkey was presented with only auditory stimuli and was required to respond to the deviant tone after its onset by a lever release. This condition will henceforth be referred to as the auditory-only condition. In condition 2, auditory and visual stimuli were presented in an interdigitated fashion, as schematically illustrated in Figure 1, and the monkey was instructed to attend to the auditory stimuli, respond to the deviant tone, and ignore all visual input. This condition will henceforth be referred to as the ignore-visual condition. In condition 3 , the monkey was again stimulated in both the auditory and visual modalities as in Figure 1 and was instructed to respond to the visual deviant and ignore all auditory input. This condition will henceforth be referred to as the attend-visual condition. A liquid reward was given to correct responses in all three conditions. If the monkey responded to a deviant in the to-be-ignored modality, the trial block was aborted; the monkey was given a brief timeout and then recued and started in a new trial block.

Data acquisition. Monkeys were surgically prepared for awake recording as described previously (Mehta et al., 2000a). LFP and MUA were sampled $(2 \mathrm{kHz})$ in $\mathrm{V} 1$ with a linear array multielectrode with 14 contacts spanning all six cortical layers (for a schematic, see Fig. $2 A$ ). The intercontact spacing was $150 \mu \mathrm{m}$. Data collection took place during periods of adequate task performance ( $>80 \%$ target detection). Multiple penetrations were made in striate cortex in each monkey. During each penetration, the multielectrode array was lowered into V1 and positioned so as to straddle its laminar depth, and allow simultaneous recording from all layers (Schroeder et al., 1998). The data set analyzed here consists of four penetrations in monkey $\mathrm{B}$ and four penetrations in monkey $\mathrm{V}$.

\section{Data analysis}

Preprocessing. The LFP data were high-pass filtered $(3 \mathrm{~Hz}$, zero phaseshift) and downsampled to $200 \mathrm{~Hz}$. For the auditory-only condition, contiguous spontaneous ongoing activity was divided into epochs of 200 $\mathrm{ms}$ in duration, which were considered trials or realizations of an under- lying stochastic process (Ding et al., 2000, 2006). For the ignore-visual and attend-visual conditions, such realizations were $200 \mathrm{~ms}$ epochs immediately preceding the onset of each visual standard stimulus (prestimulus time period), illustrated as shaded areas in Figure 1.

CSD analysis of ongoing alpha oscillatory activity. LFP distributions in the extracellular medium are generated by the first-order response to synaptic, transmembrane current flow (Mitzdorf, 1985; Schroeder et al., 1995, 1998). The second spatial derivative of LFPs yields the CSD (Mitzdorf, 1985; Schroeder et al., 1991, 1995), which allows the localization of transmembrane current generators to specific cortical layers. Because neuronal populations are stratified in the cortical layers, this in turn allows localization of transmembrane current flow components (and LFP generators) within specific cellular populations (Schroeder et al., 1998). To calculate the average current source density for ongoing oscillatory activity, the following phase-realigned averaging technique (PRAT) was used (Bollimunta et al., 2008; Chen et al., 2010). (1) The power spectrum of each recording contact for a given electrode was estimated and the contact showing the highest power spectral density at the alpha frequency $(7-13 \mathrm{~Hz})$ was chosen as the "phase index contact." (2) Sinusoid of the same frequency as the spectral peak was then fitted to the data from the phase index contact for each data epoch to obtain the phase at that frequency with respect to the beginning of the epoch. (3) The LFP data from all the contacts were shifted together according to the estimated phase at the phase index contact to realign all the trials. This step preserves the relationship among the signals from different layers. (4) The realigned data were averaged across epochs to obtain the averaged LFP for each contact. (5) A second spatial derivative was calculated from the averaged LFP profile to yield the CSD profile from which the laminar distribution of alpha current generators was identified. For ease of reference, the averaged LFP and CSD from the above procedure are referred to as PRAT-LFP and PRAT-CSD, respectively.

$C S D-M U A$ coherence. For ongoing oscillatory activity, the membrane undergoes rhythmic transition between hyperpolarization and depolarization (Lakatos et al., 2005). During the depolarizing phase of the oscillation, the pacemaker cells fire bursts of action potentials, which, via synaptic transmission, entrain neural activity in other laminae and cortical areas. Here, significant phase coherence between CSD and MUA can be used to indicate that a current generator is accompanied by rhythmic firing and thus has the potential of being a pacemaker. The MUA data were epoched the same way as the LFP data and downsampled from 2 $\mathrm{kHz}$ by taking a temporal average in nonoverlapping windows of $5 \mathrm{~ms}$ in duration to achieve effectively the same sampling resolution of $200 \mathrm{~Hz}$ as the downsampled LFPs. Three penetrations with poorly recorded MUA activity were excluded from CSD-MUA coherence analysis. For the remaining five penetrations, the coherence between single-trial CSDs around alpha current generators identified by the PRAT-CSD method and the corresponding mean-centered single-trial MUAs was calculated by the autoregressive spectral analysis method (see below). The coherence estimates were cross-checked by standard Fourier-based Matlab routines. In addition, the phase between CSD and MUA at the alpha oscillation frequency was obtained from the cross spectrum to help assess whether, during an alpha epoch, a sink (or source) corresponds with increase (or decrease) in MUA.

Spectral analysis. The logic behind our multivariate spectral analysis strategy for multielectrode laminar recordings is discussed in detail in the study by Bollimunta et al. (2008), in which the mathematical formulations for power, coherence, and Granger causality were also given. Briefly, to assess the degree of neuronal synchrony around each current generator and the interaction between current generators in different laminae, autoregressive modeling was applied, from which power, coherence, and Granger causality were obtained (Ding et al., 2000, 2006). Guided by the Akaike information criterion (Akaike, 1974), a model order of $10(50 \mathrm{~ms})$ was chosen as a trade-off between sufficient spectral resolution and over-parameterization. This model order was further verified by comparing the model-generated power spectra and the power spectra generated by Fourier-based methods. Because LFPs are recorded against a distant reference, thus susceptible to volume conduction of potentials from other sites and common reference noise, for interdependence measures such as coherence and Granger causality, single-trial 

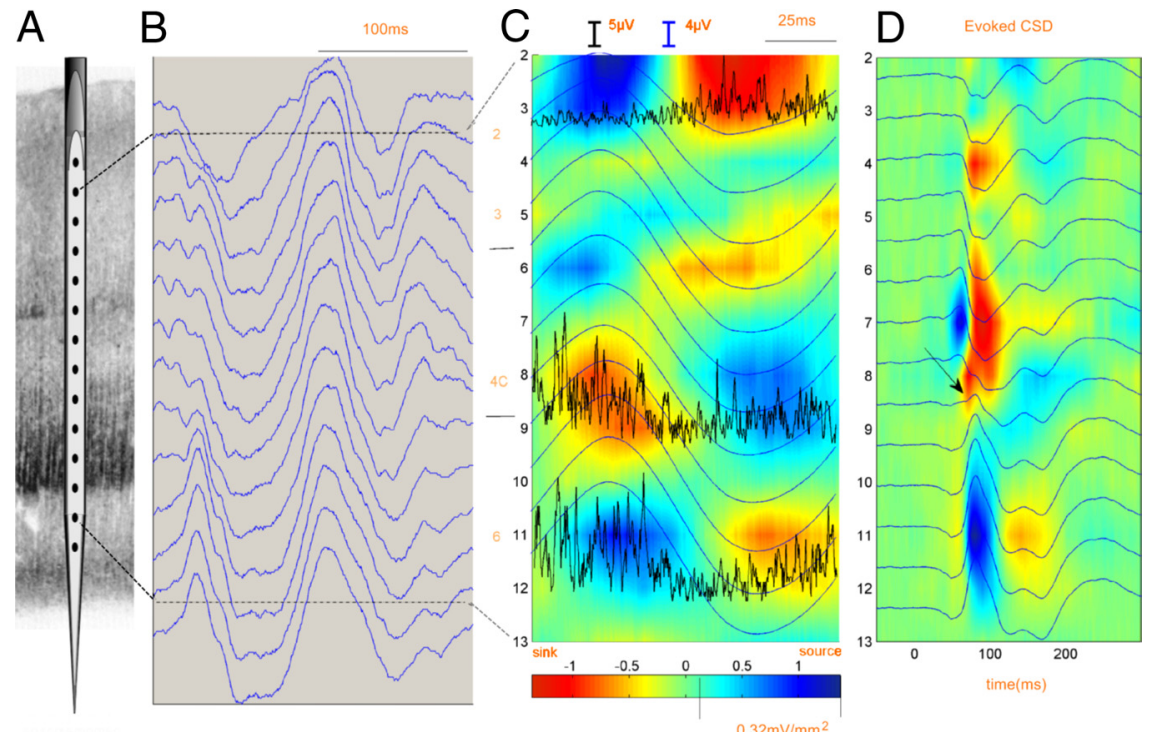

Figure 2. Alpha current generators from a typical penetration. $A$, Schematic of the multielectrode with 14 equally spaced (150 $\mu \mathrm{m}$ ) contacts. B, A short segment (200 ms) of LFPs showing alpha oscillation. C, PRAT-CSD displayed as a color-coded plot, which is the second spatial derivative of phase-realigned and averaged PRAT-LFPs (smooth blue traces). The $y$-axis is electrode contacts from 2 to 13 , with 2 being close to the cortical surface. A single epoch of MUA from three contacts is superimposed (black traces). D. Current source density profile of visual evoked activity. Laminar ERPs are overlaid (blue traces). The arrow marks the polarity inversion of the $\mathrm{N} 40$ component.
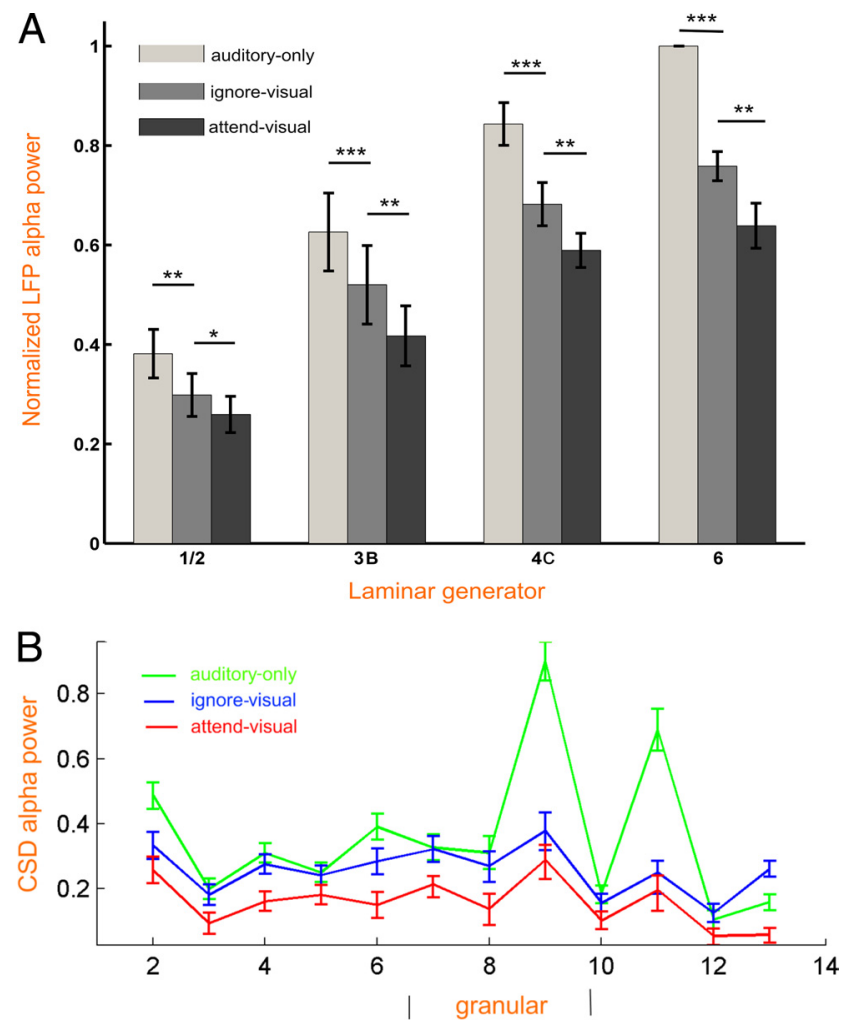

Figure 3. Attentional modulation of alpha power. $\boldsymbol{A}$, Average normalized LFP alpha peak power at the four alpha current generators located in layers $1 / 2,3 B / 4 A, 4 C$, and 6 under three experimental conditions. For each penetration, alpha peak power at each generator was divided by the alpha peak power at the layer 6 generator, and then averaged across eight penetrations. ${ }^{*} p<0.1 ;{ }^{* *} p<0.05 ;{ }^{* * *} p<0.01$. B, Averaged CSD alpha peak power as a function of recording contacts and experimental conditions. CSD alpha power exhibited similar amplitude over penetrations, and thus no normalization was performed before averaging. Error bars indicate SEM.
CSDs were obtained for each current generator and subjected to multivariate autoregressive modeling. The value of coherence ranges from 0 to 1 , with 1 indicating maximum linear interdependence and 0 indicating no linear interdependence. Granger causality is based on the concept of time series prediction (Geweke, 1982; Ding et al., 2006). For two simultaneously measured time series, one series can be called causal to the other if we can better predict the second series by incorporating past knowledge of the first one. For a cortical column containing multiple coherent alpha current generators, it has been shown that Granger causality can play a crucial role in further delineating the relationship among these generators (Bollimunta et al., 2008). In particular, primary local pacemaking generators are expected to exert causal influences on other neural ensembles.

Assessment of statistical significance. To test whether coherence or Granger causality spectra are significantly greater than zero, a random permutation approach was adopted (Brovelli et al., 2004; Bollimunta et al., 2008; Anderson et al., 2010), which built a baseline nullhypothesis distribution by assuming that the data from different trials are approximately independent of one another. Specifically, for two recording channels, trial indices are randomly shuffled to create a synthetic ensemble of trials for which there is no interdependence between the two channels, while the temporal structure within each channel is preserved. Performing such random permutations many times results in a distribution of coherence or causality spectra that corresponds to the null hypothesis of no statistical interdependence. The calculated value for a given statistic from the actual data is compared with this baseline null hypothesis distribution for the assessment of significance levels. For this work, the significance level for coherence and Granger causality was set at $p<0.001$. A Wilcoxon signed-rank test was used to evaluate the modulation of spectral quantities by different experimental conditions.

\section{Results}

Laminar profiles of LFP and MUA from the striate cortex of two macaque monkeys were analyzed. Oscillations in the alpha band $(7-13 \mathrm{~Hz})$ were observed in all eight penetrations. The average spectral peak frequency is $8.9 \pm 1.2 \mathrm{~Hz}$ based on LFP signals and $9.5 \pm 1.9 \mathrm{~Hz}$ based on CSD signals.

\section{Laminar distribution of the alpha current generators}

Figure $2 B$ depicts a 200 ms epoch of LFP data from a representative penetration in V1 under the auditory-only condition. Two full cycles of rhythmic oscillations around $10 \mathrm{~Hz}$ are clearly seen. The CSD profile accompanying this oscillatory activity was obtained by the PRAT method and shown in Figure $2 C$ together with the PRAT-LFPs. Here, contact 12 has the highest spectral power and was thus used as the phase index contact. Also included in Figure $2 C$ are MUA signals from a subset of recording contacts. Four alpha current generators with temporally alternating current sources (blue) and sinks (red) are readily identified in the supragranular (SG), granular (G), and the infragranular (IG) layers. The IG layer alpha current generator is located approximately in layer 6 (Fig. 2C). The G layer alpha current generator is located approximately in layer $4 \mathrm{C}$ and is out of phase with the layer 6 generator. The SG alpha current generator is located approximately in layers $1 / 2$ and is in phase with the layer 6 genera- 
tor. A weak alpha current generator is also seen around lower layer $3 \mathrm{~B}$ and upper layer 4A (Fig. 2C). No polarity inversion is seen in the PRAT-LFPs in Figure $2 C$ and the current sinks (red) at layers $6,3 \mathrm{~B} / 4 \mathrm{~A}$, $1 / 2$ are associated with negative PRATLFPs. Similar laminar PRAT-CSD characteristics were found in other penetrations (supplemental Fig. 1, available at www. jneurosci.org as supplemental material). Different experimental conditions modulated the amplitude of alpha oscillations but not the laminar distribution of the current generators (see below).

In addition to ongoing oscillations, the CSD profiles of the stimulus-evoked activity, together with the event-related potentials (ERPs), are shown in Figure 2D for comparison. The polarity inversion of the N40 ERP component and the accompanying current sink in the granular layers (Fig. 2D, arrow) helped to determine the boundary between layer 4C and layer 5 (Schroeder at al., 1995, 1998; Mehta et al., 2000a).

\section{Attentional modulation of alpha power}

For both attend-visual and ignore-visual conditions, the power spectrum was estimated for the data from the $200 \mathrm{~ms}$ interval immediately preceding the onset of the visual standard stimulus (Fig. 1, shaded areas) (see Materials and Methods). For the auditory-only condition, data were divided into epochs of $200 \mathrm{~ms}$ in duration, and then submitted to spectral analysis. Each of the four alpha current generators was represented by LFP from a single contact. For example, for the penetration in Figure 2, contacts 2, 6, 8, and 11 represented alpha current generators in layers $1 / 2,3 \mathrm{~B} / 4 \mathrm{~A}, 4 \mathrm{C}$, and 6. LFP peak alpha power from all generators was normalized by auditory-only alpha peak power at layer 6 and averaged across all eight penetrations $(n=8)$ (Fig. $3 A)$. For both attend-visual and ignore-visual conditions, alpha power decreased significantly relative to the auditory-only condition $(p<0.05)$, with the largest decrease occurring over lower G and IG layers. On switching attention from the auditory to the visual modality, alpha power exhibited additional significant decline $(p<0.05$ for $3 \mathrm{~B} / 4 \mathrm{~A}, 4 \mathrm{C}$, and layer 6 generators; $p<0.1$ for layer $1 / 2$ generator).

To further localize the effect of attention, single-trial CSD profiles were derived, and CSD alpha peak power was averaged $(n=8)$ and plotted as a function of recording contacts for all three experimental conditions in Figure $3 B$. When the ignorevisual condition was compared with the auditory-only condition, a large reduction $(>50 \%)$ of alpha power was seen over lower $\mathrm{G}$ (layer 4C) and IG layers (layer 6), consistent with the subsequent finding that these layers contain the local primary pacemaking alpha generators (see below). Switching attention from the auditory to the visual domain (i.e., under the attend-visual condition) led to an additional decrease of $30 \%$ in alpha power, and this decrease was not layer specific (Fig. $3 B$, red curve relative to blue curve). Figure 2.
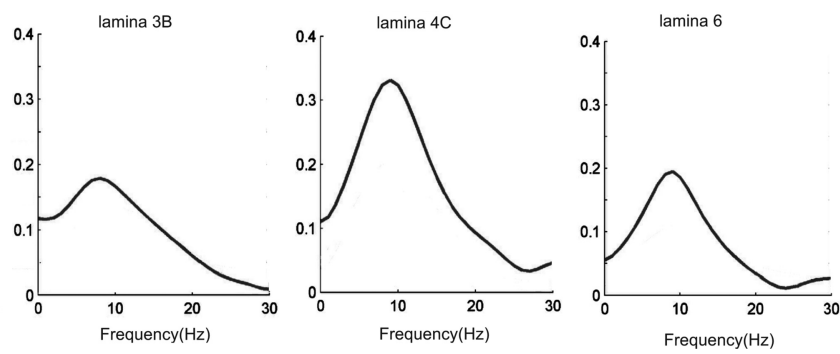

Figure 4. CSD-MUA coherence at the four alpha current generators identified by PRAT-CSD for the penetration shown in
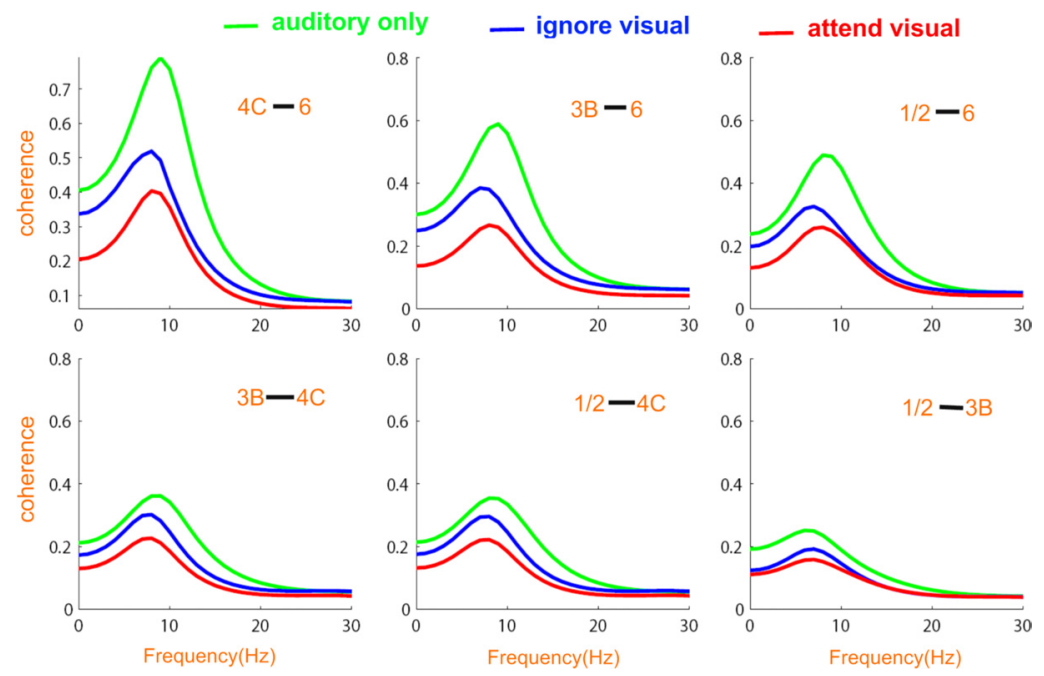

Figure 5. Pairwise CSD coherence spectra among the four alpha generators under three experimental conditions for the penetration shown in Figure 2.

\section{CSD-MUA coherence}

For each channel, the CSD and MUA data were subjected to autoregressive modeling from which the CSD-MUA coherence was derived to assess whether the firing probability of the local neurons around a given alpha current generator is phase-locked to the underlying oscillation, a necessary condition for pacemaking. For all the alpha current generators identified in Figure 2C, CSD-MUA coherence was significant, as shown in Figure 4 . Across five penetrations $(n=5)$, the average peak CSD-MUA coherence in the alpha range was 0.19 in layer $6,0.33$ in layer $4 \mathrm{C}, 0.17$ in layer $3 \mathrm{~B} / 4 \mathrm{~A}$, and 0.17 in layer $1 / 2$.

The relative phase between CSD and MUA was computed to evaluate the relationship between firing probability and the temporal sink-source organization of the alpha generator. The phase spectrum was found to be continuous in the alpha range with a mean $(n=5)$ phase difference of $16.0 \pm 6.4^{\circ}$ at layer 6 , $167.0 \pm 7.2^{\circ}$ at layer $4 \mathrm{C}, 170.0 \pm 5.4^{\circ}$ at layer $3 \mathrm{~B} / 4 \mathrm{~A}$, and $163.0 \pm 12.4^{\circ}$ at layer $1 / 2$. The phase relationship between CSD and MUA at layers $4 \mathrm{C}, 3 \mathrm{~B} / 4 \mathrm{~A}$, and $1 / 2$ suggests that the increased action potential firing in $\mathrm{G}$ and $\mathrm{SG}$ layers coincide with alpha current sinks. At layer 6, however, the near inphase relationship indicates that the current source is accompanied by an increase in MUA. A likely explanation is that the recorded MUA at layer 6 contained a strong contribution from the depolarization of presynaptic thalamocortical axons (Tenke et al., 1993; Schroeder et al., 1998; Swadlow et al., 2002). 
Table 1. Alpha peak coherence values for three experimental conditions for every penetration and every pair of alpha current generators

\begin{tabular}{|c|c|c|c|c|c|c|c|c|}
\hline Penetration & 1 & 2 & 3 & 4 & 5 & 6 & 7 & 8 \\
\hline \multicolumn{9}{|l|}{$6-4 C$} \\
\hline Auditory only & $0.79(9)$ & $0.65(10)$ & $0.24(9)$ & $0.58(11)$ & $0.44(9)$ & $0.73(9)$ & $0.55(10)$ & $0.65(9)$ \\
\hline Ignore visual & $0.52(9)$ & $0.44(10)$ & $0.13(9)$ & $0.35(10)$ & $0.34(8)$ & $0.50(9)$ & $0.30(10)$ & $0.51(9)$ \\
\hline Attend visual & $0.40(9)$ & $0.30(9)$ & $0.08(8)$ & $0.24(10)$ & $0.30(10)$ & $0.30(9)$ & $0.25(9)$ & $0.25(10)$ \\
\hline \multicolumn{9}{|l|}{$6-3 B$} \\
\hline Auditory only & $0.59(9)$ & $0.65(11)$ & $0.34(10)$ & $0.52(9)$ & $0.49(10)$ & $0.43(9)$ & $0.42(10)$ & $0.39(10)$ \\
\hline Ignore visual & $0.39(9)$ & $0.40(10)$ & $0.20(10)$ & $0.37(10)$ & $0.33(10)$ & $0.22(9)$ & $0.26(10)$ & $0.39(10)$ \\
\hline Attend visual & $0.26(8)$ & $0.24(10)$ & $0.16(10)$ & $0.19(10)$ & $0.21(9)$ & $0.22(9)$ & $0.18(10)$ & $0.35(10)$ \\
\hline \multicolumn{9}{|l|}{$6-1 / 2$} \\
\hline Auditory only & $0.49(8)$ & $0.55(10)$ & $0.23(9)$ & $0.19(9)$ & $0.44(8)$ & $0.29(8)$ & $0.10(7)$ & $0.33(10)$ \\
\hline Ignore visual & $0.32(7)$ & $0.33(9)$ & $0.24(9)$ & $0.10(9)$ & $0.30(8)$ & $0.23(8)$ & $0.9(8)$ & $0.20(9)$ \\
\hline Attend visual & $0.26(8)$ & $0.25(8)$ & $0.18(8)$ & $0.10(9)$ & $0.25(8)$ & $0.20(7)$ & $0.11(8)$ & $0.11(9)$ \\
\hline \multicolumn{9}{|l|}{$4 C-3 B$} \\
\hline Auditory only & $0.36(9)$ & $0.40(9)$ & $0.30(10)$ & $0.15(9)$ & $0.33(8)$ & $0.18(9)$ & $0.22(8)$ & $0.36(10)$ \\
\hline Ignore visual & $0.30(8)$ & $0.41(9)$ & $0.20(9)$ & $0.06(8)$ & $0.18(8)$ & $0.11(9)$ & $0.12(7)$ & $0.22(9)$ \\
\hline Attend visual & $0.22(8)$ & $0.30(9)$ & $0.21(9)$ & $0.04(7)$ & $0.10(8)$ & $0.10(9)$ & $0.08(7)$ & $0.18(9)$ \\
\hline \multicolumn{9}{|l|}{$4 C-1 / 2$} \\
\hline Auditory only & $0.35(10)$ & $0.42(10)$ & $0.14(8)$ & $0.23(10)$ & $0.49(8)$ & $0.31(8)$ & $0.15(7)$ & $0.22(10)$ \\
\hline Ignore visual & $0.29(8)$ & $0.37(9)$ & $0.13(8)$ & $0.18(9)$ & $0.40(8)$ & $0.15(8)$ & $0.16(8)$ & $0.24(10)$ \\
\hline Attend visual & $0.22(9)$ & $0.33(9)$ & $0.05(7)$ & $0.18(9)$ & $0.33(8)$ & $0.14(8)$ & $0.14(8)$ & $0.24(10)$ \\
\hline \multicolumn{9}{|l|}{$3 B-1 / 2$} \\
\hline Auditory only & $0.25(7)$ & $0.15(9)$ & $0.11(8)$ & $0.20(9)$ & $0.25(8)$ & $0.11(6)$ & $0.19(8)$ & $0.24(10)$ \\
\hline Ignore visual & $0.19(7)$ & $0.15(9)$ & $0.09(7)$ & $0.17(9)$ & $0.19(8)$ & $0.06(7)$ & $0.22(8)$ & $0.18(9)$ \\
\hline Attend visual & $0.16(7)$ & $0.15(9)$ & $0.10(7)$ & $0.05(8)$ & $0.13(8)$ & $0.08(7)$ & $0.15(8)$ & $0.15(9)$ \\
\hline
\end{tabular}

Table 2. Average percentage changes in alpha peak coherence between experimental conditions for different generator pairs

\begin{tabular}{lllllll}
\hline & \multicolumn{1}{l}{ Pairs } \\
\cline { 2 - 6 } Conditions & $6-4 \mathrm{C}$ & $6-3 \mathrm{~B}$ & $6-1 / 2$ & $4 \mathrm{C}-3 \mathrm{~B}$ & $4 \mathrm{C}-1 / 2$ & $3 \mathrm{~B}-1 / 2$ \\
\hline IV-A0 & $-34 \%^{* * * *}$ & $-33 \%^{* * *}$ & $-27 \%^{* *}$ & $-35 \%^{* *}$ & $-14 \%^{*}$ & $-17 \%^{*}$ \\
$\mathrm{AV}-\mathrm{IV}$ & $-30 \%^{* * *}$ & $-27 \%^{* * *}$ & $-15 \%^{*}$ & $-23 \%^{* *}$ & $-17 \%^{* *}$ & $-15 \%$ \\
\hline
\end{tabular}

A0, Auditory only; AV, attend visual; IV, ignore visual.

${ }^{*} p<0.1 ;{ }^{* *} p<0.01 ;{ }^{* * *} p<0.005$.

\section{Interaction of alpha current generators and attentional modulation}

The relationship between alpha current generators was assessed by applying coherence and Granger causality analysis to single-trial CSDs derived from LFP data. For the penetration in Figure 2, the layer 6 alpha generator is represented by CSD at contact 11, the layer $4 \mathrm{C}$ generator at contact 8 , the layer $3 \mathrm{~B} / 4 \mathrm{~A}$ generator at contact 6 , and the layer $1 / 2$ generator at contact 2 . These four CSD signals were then subjected to autoregressive modeling. Statistical significance was assessed by a random permutation test, and the significance level was set at $p<0.001$ (see Materials and Methods).

For the penetration in Figure 2, the coherence spectra were obtained for all distinct pairs of alpha current generators and shown for the three experimental conditions in Figure 5. Relative to the auditory-only condition, the alpha coherence decreased for the ignore-visual condition, and an additional decline in alpha coherence was seen when attention was switched from the auditory domain to the visual domain. Table 1 summarizes the results for all eight penetrations where the peak alpha coherence for different alpha generator pairs is given for the three experimental conditions (numbers in parentheses indicating peak frequencies). The average percentage changes of alpha coherence between ignore-visual and auditory-only conditions and between attend-visual and ignorevisual conditions are summarized in Table 2, where the statistical significance of the changes is indicated. From Tables 1 and 2, it can be seen that the pattern observed in Figure 5 is consistent across penetrations.
The synchronized alpha activity between generators was further decomposed by Granger causality into its directional components. For the penetration in Figure 2, a significant peak in the alpha range with a peak value of 0.38 is seen for $6 \rightarrow 4 \mathrm{C}$ (Fig. $6 A$ ) under the auditory-only condition, and the alpha peak in the opposite direction $(4 \mathrm{C} \rightarrow 6)$ was also significant with a value of 0.21 , suggesting that the alpha generators at layer 6 and $4 \mathrm{C}$ are reciprocally coupled. This pattern of bidirectional driving was consistent across all eight penetrations as detailed in Table 3.

Also in Figure $6 \mathrm{~A}$, the layer 6 generator drives layer $3 \mathrm{~B} / 4 \mathrm{~A}$ and $1 / 2$ generators, with significant peak causality values of 0.18 at 10 $\mathrm{Hz}$ for $6 \rightarrow 3 \mathrm{~B} / 4 \mathrm{~A}$, and 0.51 at $8 \mathrm{~Hz}$ for $6 \rightarrow 1 / 2$. Across penetrations, $6 \rightarrow 1 / 2$ is significant for all eight penetrations (Table 3 ), and $6 \rightarrow 3 \mathrm{~B} / 4 \mathrm{~A}$ is significant in five penetrations (Table 3 ). Similarly, layer $4 \mathrm{C}$ drives layer $3 \mathrm{~B} / 4 \mathrm{~A}$ and $1 / 2$, with the peak causality value of 0.20 at $10 \mathrm{~Hz}$ for $4 \mathrm{C} \rightarrow 3 \mathrm{~B} / 4 \mathrm{~A}$, and 0.52 at $8 \mathrm{~Hz}$ for $4 \mathrm{C} \rightarrow 1 / 2$. This pattern is consistent across all eight penetrations (Table 4). For the Granger causality in the opposite directions, $1 / 2 \rightarrow 4 \mathrm{C}, 1 / 2 \rightarrow 6,3 \mathrm{~B} / 4 \mathrm{~A} \rightarrow 4 \mathrm{C}$, and $3 \mathrm{~B} / 4 \mathrm{~A} \rightarrow 6$, the values were not consistently significant across penetrations (Tables 3,4$)$. In Figure $6 A$, the $3 \mathrm{~B} / 4 \mathrm{~A} \rightarrow 1 / 2$ had a significant peak value of 0.15 at 10 $\mathrm{Hz}$. However, after conditioning out layer 6 activities (Chen et al., 2006; Ding et al., 2006; Bollimunta et al., 2008), the peak value was reduced to 0.01 , which is no longer statistically significant. A possible explanation is that layer 6 generator drives layer $3 \mathrm{~B} / 4 \mathrm{~A}$ generator with shorter delays than it does layer $1 / 2$ generator. This can lead to false causal influences from the $3 \mathrm{~B} / 4 \mathrm{~A}$ generator to the $1 / 2$ generator under pairwise analysis (Kaminski et al., 2001). The Granger causality in the opposite direction, $1 / 2 \rightarrow 3 \mathrm{~B} /$ $4 \mathrm{~A}$, is not significant. In Figure $6 \mathrm{~B}$, the pattern of interaction among the alpha current generators in $\mathrm{V} 1$ is schematically represented, where an arrow is plotted between two generators if more than one-half of the penetrations $(n>4)$ exhibit significant alpha Granger causality in that direction.

Relative to the auditory-only condition, Granger causality values declined significantly for both ignore-visual and attendvisual conditions, with the attend-visual condition showing 
additional decline from the ignore-visual condition (Tables 3,4 ). Table 5 summarizes the average percentage changes of alpha Granger causality for different generator pairs. It is worth noting that the Granger causality decrease does not exhibit obvious layer specificity, suggesting that the intralaminar circuitry of the alpha rhythm does not change qualitatively as a result of task performance or attention. These results, together with the results on the attentional modulation of alpha power and coherence, suggest that the decrease in alpha activity occurs both at the level of local neuronal ensembles (e.g., a given layer) and at the level of their interactions in local circuits (e.g., between layers).

\section{Discussion}

We investigated the neuronal mechanisms and attentional modulation of alpha oscillations $(\sim 10 \mathrm{~Hz})$ in macaque primary visual cortex by analyzing laminar profiles of local field potential and multiunit activity. There are three main results. First, multiple, separable alpha current generators were identified in SG,
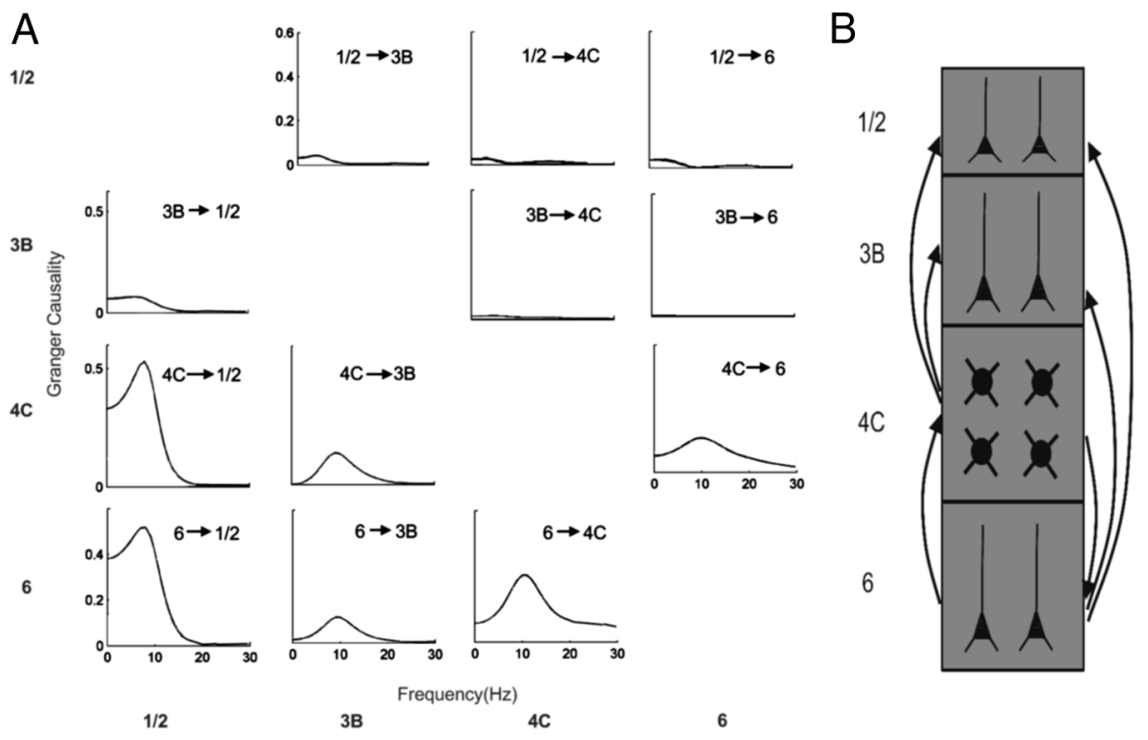

Figure 6. Granger causality analysis. $\boldsymbol{A}$, Granger causality spectra for different alpha current generator pairs for the penetration in Figure 2. The $y$-axis is the driver and $x$-axis is the target (i.e., panel $x y$, where $x$ is the row index of the panel and $y$ is the column index, shows the Granger causality spectrum for $y \rightarrow x$ ). $\boldsymbol{B}$, Schematic representation of interaction between different alpha current generators. An arrow, understood in the sense of Granger causality, is plotted between two alpha current generators if more than one-half of the penetrations $(n>4)$ showed significant alpha Granger causality in that direction under the auditory-only condition. consistently the strongest across penetrations. Second, alpha current generators in different cortical layers are synchronized, with the $4 \mathrm{C}$ generator and the layer 6 generator reciprocally driving each other and both driving the generators in other layers. Third, attention reduces alpha power, with the strongest reduction seen over lower $G$ and IG layers, and attention also reduces alpha coherence and Granger causality, with no obvious layer specific effects. We note that after the completion of this work a report appeared in which distinct laminar patterns of ongoing LFP activity including alpha oscillations are found in macaque V1 (Maier et al., 2010).

\section{Thalamocortical genesis of alpha rhythm in V1}

Local field potential oscillations reflect rhythmic current flows across cellular membranes. In this sense, all alpha current generators in Figure 2 contribute to cortical alpha rhythm sampled by extracellular electrodes. The significant CSD-MUA coherence at these generators suggests that the membrane oscillations in the neuronal ensemble underlying each generator are suprathreshold and are accompanied by rhythmic firing. Among the four potential pacemaker generators, Granger causality analysis implicated the layer $4 \mathrm{C}$ and the layer 6 generators as primary local pacemaking generators. This result seems to be consistent with the idea that the alpha rhythm in striate cortex is the consequence of thalamocortical interaction in the form of $\mathrm{V} 1$ layer $6 \rightarrow \mathrm{LGN} \rightarrow \mathrm{V} 1$ layer $4 \mathrm{C} \rightarrow \mathrm{V} 1$ layer 6 .

The thalamus has long been associated with the generation of cortical oscillations (Andersen and Andersson, 1968; Destexhe and Sejnowski, 2001). Recent in vitro and in vivo studies show that a group of core cells in the lateral geniculate nucleus are capable of exhibiting alpha frequency oscillations under cholinergic activation (Hughes et al., 2004; Lörincz et al., 2008). Given that the LGN core cells predominately project to the granular layers, particularly layer 4C, of the cortex (Jones, 2001), the 4C generator in Figure 2 is likely the cortical recipient of any driving

thalamocortical alpha input. In parallel to these developments in the thalamic genesis of alpha, in vivo recordings in dogs (Lopes da Silva et al., 1973a,b), as well as in vitro slice preparations (Silva et al., 1991), have identified IG layers, particularly layer 5, as containing pacemaker neurons in alpha frequencies. This finding has led to computational models placing alpha pacemakers in layer 5 (Jones et al., 2000; Karameh et al., 2006). In behaving monkeys, Bollimunta et al. (2008) showed that, in extrastriate cortical areas $\mathrm{V} 2$ and V4, the alpha current in layer 5 is the primary local pacemaker. Although the IG alpha current generator in the present work appears to be in layer 6 , which is the main cortical layer that projects back to LGN, the contributions from layer 5 neurons cannot be ruled out.

In the context of the foregoing, we make three additional observations. First, an alpha current generator is also found in layer 4 of V2 and V4 (Bollimunta et al., 2008), but Granger causality analysis indicates that this generator is not a primary local pacemaker and is unidirectionally driven by the primary local pacemaker generator in IG layers, in contrast to what we found in V1. This difference may highlight the fact that only the striate cortex receives driving input from the LGN principal cells in primates (Jones, 2001, 2002). The thalamic projections to extrastriate cortex come from nonspecific thalamic neurons and higher order nuclei such as the pulvinar, and target extragranular (mainly SG) layers (Sherman and Guillery, 2001). Given that the feedback from the striate cortex to LGN principal cells originates from layer 6 of the same cortical column to which they send afferents (Callaway, 1998; Jones, 2002; Sherman and Guillery, 2002), the V1 alpha appears to be generated by thalamocortical interaction, whereas in the higher order cortical areas such as V2 and V4, alpha is likely generated cortically, which is then modulated and possibly also driven by influences from the thalamus. Second, using lesion and physiological recording methods, Silva et al. (1991) have shown that, in cortical slices from the rat primary somatosensory cortex $S 1$, the pacemaker cells are located in layer 5 . This finding, together with the data 
Table 3. Alpha peak Granger causality values for generator pairs involving layer 6 for three experimental conditions

\begin{tabular}{|c|c|c|c|c|c|c|c|c|}
\hline Penetration & 1 & 2 & 3 & 4 & 5 & 6 & 7 & 8 \\
\hline \multicolumn{9}{|l|}{$6 \rightarrow 4 C$} \\
\hline Auditory only & $0.38(10)$ & $0.42(10)$ & $0.14(9)$ & $0.53(10)$ & $0.49(10)$ & $0.23(8)$ & $0.39(10)$ & $0.47(9)$ \\
\hline Ignore visual & $0.32(10)$ & $0.27(10)$ & $0.11(10)$ & $0.38(10)$ & $0.44(10)$ & $0.22(8)$ & $0.36(10)$ & $0.44(10)$ \\
\hline Attend visual & $0.27(10)$ & $0.25(10)$ & $0.10(10)$ & $0.28(10)$ & $0.34(10)$ & $0.29(9)$ & $0.24(10)$ & $0.40(9)$ \\
\hline \multicolumn{9}{|l|}{$4 C \rightarrow 6$} \\
\hline Auditory only & $0.21(10)$ & $0.44(10)$ & $0.23(11)$ & $0.22(9)$ & $0.38(10)$ & $0.33(9)$ & $0.52(10)$ & $0.44(11)$ \\
\hline Ignore visual & $0.18(10)$ & $0.30(9)$ & $0.12(10)$ & $0.17(10)$ & $0.37(10)$ & $0.28(10)$ & $0.36(10)$ & $0.40(10)$ \\
\hline Attend visual & $0.18(10)$ & $0.23(10)$ & $0.09(10)$ & $0.18(10)$ & $0.28(9)$ & $0.20(10)$ & $0.30(10)$ & $0.40(10)$ \\
\hline \multicolumn{9}{|l|}{$6 \rightarrow 3 \mathrm{~B}$} \\
\hline Auditory only & $0.18(10)$ & $0.32(10)$ & $0.44(9)$ & NS & NS & $0.52(10)$ & NS & $0.63(11)$ \\
\hline Ignore visual & $0.12(10)$ & $0.24(10)$ & $0.35(10)$ & NS & NS & $0.44(10)$ & NS & $0.52(10)$ \\
\hline Attend visual & $0.08(10)$ & $0.15(10)$ & $0.33(10)$ & NS & NS & $0.35(9)$ & NS & $0.51(10)$ \\
\hline \multicolumn{9}{|l|}{$3 \mathrm{~B} \rightarrow 6$} \\
\hline Auditory only & NS & NS & $0.10(10)$ & NS & NS & $0.18(8)$ & NS & $0.06(10)$ \\
\hline Ignore visual & NS & NS & NS & NS & NS & $0.07(9)$ & NS & NS \\
\hline Attend visual & NS & NS & NS & NS & NS & NS & NS & NS \\
\hline \multicolumn{9}{|l|}{$6 \rightarrow 1 / 2$} \\
\hline Auditory only & $0.51(8)$ & $0.32(10)$ & $0.44(9)$ & $0.53(10)$ & $0.59(10)$ & $0.33(8)$ & $0.29(10)$ & $0.37(10)$ \\
\hline Ignore visual & $0.44(8)$ & $0.27(10)$ & $0.31(10)$ & $0.38(10)$ & $0.46(10)$ & $0.22(8)$ & $0.26(10)$ & $0.34(10)$ \\
\hline Attend visual & $0.35(9)$ & $0.15(10)$ & $0.30(10)$ & $0.28(10)$ & $0.34(10)$ & $0.19(9)$ & $0.24(10)$ & $0.30(10)$ \\
\hline \multicolumn{9}{|l|}{$1 / 2 \rightarrow 6$} \\
\hline Auditory only & NS & $0.10(10)$ & $0.13(11)$ & $0.12(9)$ & NS & NS & NS & $0.34(10)$ \\
\hline Ignore visual & NS & $0.05(10)$ & $0.10(10)$ & $0.07(10)$ & NS & NS & NS & $0.20(10)$ \\
\hline Attend visual & NS & NS & $0.06(10)$ & NS & NS & NS & NS & $0.18(10)$ \\
\hline
\end{tabular}

Table 4. Alpha peak Granger causality values for generator pairs involving layer $4 \mathrm{C}$ for three experimental conditions

\begin{tabular}{|c|c|c|c|c|c|c|c|c|}
\hline Penetration & 1 & 2 & 3 & 4 & 5 & 6 & 7 & 8 \\
\hline \multicolumn{9}{|l|}{$4 C \rightarrow 1 / 2$} \\
\hline Auditory only & $0.52(8)$ & $0.42(10)$ & $0.24(9)$ & $0.45(10)$ & $0.39(10)$ & $0.30(10)$ & $0.19(10)$ & $0.47(10)$ \\
\hline Ignore visual & $0.46(10)$ & $0.33(10)$ & $0.11(10)$ & $0.39(10)$ & $0.36(10)$ & $0.32(10)$ & $0.16(10)$ & $0.24(10)$ \\
\hline Attend visual & $0.37(10)$ & $0.25(10)$ & $0.10(10)$ & $0.30(10)$ & $0.34(10)$ & $0.29(11)$ & $0.08(10)$ & $0.20(10)$ \\
\hline \multicolumn{9}{|l|}{$1 / 2 \rightarrow 4 C$} \\
\hline Auditory only & NS & NS & $0.18(11)$ & NS & $0.12(10)$ & NS & $0.15(9)$ & $0.19(9)$ \\
\hline Ignore visual & NS & NS & $0.17(10)$ & NS & NS & NS & NS & $0.10(8)$ \\
\hline Attend visual & NS & NS & $0.10(10)$ & NS & NS & NS & NS & NS \\
\hline \multicolumn{9}{|l|}{$4 C \rightarrow 3 B$} \\
\hline Auditory only & $0.20(10)$ & $0.43(10)$ & $0.52(9)$ & $0.33(10)$ & $0.39(10)$ & $0.43(8)$ & $0.24(10)$ & $0.37(10)$ \\
\hline Ignore visual & $0.14(10)$ & $0.33(10)$ & $0.44(10)$ & $0.28(10)$ & $0.26(10)$ & $0.28(8)$ & $0.24(10)$ & $0.34(10)$ \\
\hline Attend visual & $0.15(10)$ & $0.25(10)$ & $0.38(10)$ & $0.28(10)$ & $0.24(10)$ & $0.29(9)$ & $0.22(10)$ & $0.30(10)$ \\
\hline \multicolumn{9}{|l|}{$3 B \rightarrow 4 C$} \\
\hline Auditory only & $0.05(9)$ & NS & NS & NS & $0.11(9)$ & NS & $0.14(10)$ & NS \\
\hline Ignore visual & NS & NS & NS & NS & $0.10(9)$ & NS & $0.10(10)$ & NS \\
\hline Attend visual & NS & NS & NS & NS & $0.05(8)$ & NS & NS & NS \\
\hline
\end{tabular}

Table 5. Average percentage changes in alpha peak Granger causality between experimental conditions for different generator pairs

\begin{tabular}{|c|c|c|c|c|c|c|}
\hline \multirow[b]{2}{*}{ Conditions } & \multicolumn{6}{|l|}{ Pairs } \\
\hline & $6 \rightarrow 4 C$ & $6 \rightarrow 3 B$ & $6 \rightarrow 1 / 2$ & $4 C \rightarrow 6$ & $4 C \rightarrow 3 B$ & $4 C \rightarrow 1 / 2$ \\
\hline IV-A0 & $-16 \%$ ** & $-22 \% *$ & $-20 \% * *$ & $-22 \% * *$ & $-20 \% *$ & $-21 \%^{*}$ \\
\hline AV-IV & $-11 \% *$ & $-20 \% *$ & $-19 \% * *$ & $-14 \% *$ & $-7 \% *$ & $-20 \% *$ \\
\hline
\end{tabular}

A0, Auditory only; AV, attend visual; IV, ignore visual.

${ }^{*} p<0.1 ;{ }^{* *} p<0.01$

presented here, suggests that layer 4 "granular" neurons may not be endowed with membrane properties that cause them to oscillate intrinsically. In the absence of thalamic input, their oscillatory activity mainly comes from the driving input of the deep layer pacemaker neurons. Third, it is important to note that our inference of the thalamocortical genesis of V1 alpha relies mainly on data from $\mathrm{V} 1$ and extant literature. In the absence of strong simultaneous LGN recordings, this infer- ence remains indirect, and the possibility of a cortical genesis of V1 alpha cannot be ruled out.

Attentional modulation of alpha activity

How attention modulates alpha has been extensively studied (Shaw, 2003; Palva and Palva, 2007). An emerging consensus is that, when attention is directed externally (sensory intake), the magnitude of alpha decreases. In contrast, when attention is directed internally (sensory rejection) (e.g., during retention of working memory), alpha magnitude increases. Physiologically, the suppression of alpha is thought to reflect increased cortical excitability (Jones et al., 2000; Worden et al., 2000; Klimesch et al., 2007; Romei et al., 2008; Rajagovindan and Ding, 2010), whereas the enhancement of alpha is thought to reflect decreased excitability in task-irrelevant brain areas to prevent task-relevant information maintained working memory buffer from interference (Jensen et al., 2002). Consistent with these ideas, alpha power in the striate cortex, which is primarily involved in sensory 
detection and encoding, was found to decline significantly with visual attention, relative to when the monkey attends auditory stimuli during bimodal stimulation. In addition, coherence and Granger causality in the alpha range also decreased with visual attention, suggesting that the alpha decline recorded on the scalp could be the consequence of desynchronization at both the level of a local neuronal ensemble and that of the columnar circuit. The interaction pattern between alpha current generators, however, remains qualitatively the same between experimental conditions, indicating that attention does not alter the laminar organization of alpha activity, only the magnitude.

Three additional observations merit emphasis. First, compared with the auditory-only condition, both ignore-visual and attend-visual conditions exhibited decrease in alpha power, coherence, and Granger causality. This decrease likely mainly reflects an increased level of cortical arousal attributable to bimodal stimulation. The additional decline of alpha activity in the attend-visual condition relative to the ignore-visual condition is likely the consequence of selective visual attention because approximately the same level of arousal between the two conditions, measured in terms of task performance, is maintained. Second, in addition to the principal (core) neurons in the LGN, which are thought to provide the driving input to the layer $4 \mathrm{C}$ alpha generator, the so-called "koniocellular" (matrix) neurons, which project predominantly to the superficial layers of the cortex, may also play a modulatory role on cortical alpha activity. For two penetrations in monkey B where simultaneous LGN and V1 recordings are available, a coherence analysis reveals significant alpha coherence between LGN and the superficial layers of V1, which is reduced with the deployment of visual attention (supplemental Fig. 2, available at www.jneurosci.org as supplemental material). Third, $\sim 10 \mathrm{~Hz}$ oscillations in the somatosensory cortex are considered in recent work, and they are found to be modulated by behavioral state (Jones et al., 2010; Zhang and Ding, 2010). Moreover, in studies of the anesthetic agent propofol, alpha activity has been used as an index of the level of consciousness (Feshchenko et al., 2004). The underlying thalamocortical mechanisms are explored in a computational model (Ching et al., 2010).

\section{Granger causality and hypothesis testing}

Synchronous alpha oscillations between the thalamus and the cortex have been reported in a number of in vivo experiments (Lopes da Silva et al., 1973b; Rougeul-Buser and Buser, 1997). Depending on the hypothesized role played by LGN and V1, one may make different predictions regarding the laminar profile of alpha current generators and their patterns of interaction. If alpha rhythm is of a cortical origin with deep-layer pyramidal neurons acting as pacemakers, then the deep-layer generator is expected to unidirectionally drive the generators in other layers. However, if alpha rhythm is of a thalamic origin, then the generator in $4 \mathrm{C}$, on receiving the driving input from the LGN core cells, is expected to exert unidirectional causal influences on other generators. Finally, if alpha rhythm is the consequence of thalamocortical interaction, then layer 4C and deep layer generators will drive each other and they both will drive the generators in other layers. Our results, summarized in Figure $6 B$, appear to favor the thalamocortical genesis of alpha rhythm in V1. Additional delineation of the role played by each structure in the thalamocortical loop, however, is beyond the capacity of the present analysis framework. To illustrate, consider two neuronal ensembles A and B. Suppose that pacemaker cells are contained in ensemble A but not in ensemble B. If Granger causal influences between A and B are reciprocal, then in the absence of additional information, it is likely that we will not be able to ascertain in this closed loop where the rhythm originates.

\section{References}

Akaike H (1974) A new look at the statistical model identification. IEEE Trans Automat Control 19:716-723.

Andersen P, Andersson SA (1968) Physiological basis of the alpha rhythm. New York: Appleton-Century-Crofts.

Anderson KL, Rajagovindan R, Ghacibeh GA, Meador KJ, Ding M (2010) Theta oscillations mediate interaction between prefrontal cortex and medial temporal lobe in human memory. Cereb Cortex 20:1604-1612.

Bollimunta A, Chen Y, Schroeder CE, Ding M (2008) Neuronal mechanisms of cortical alpha oscillations in awake-behaving macaques. J Neurosci 28:9976-9988.

Brovelli A, Ding M, Ledberg A, Chen Y, Nakamura R, Bressler SL (2004) Beta oscillations in a large-scale sensorimotor cortical network: directional influences revealed by Granger causality. Proc Natl Acad Sci U S A 101:9849-9854

Buzsaki G (2006) Rhythms of the brain. New York: Oxford UP.

Callaway EM (1998) Local circuits in primary visual cortex of the macaque monkey. Annu Rev Neurosci 21:47-74.

Castro-Alamancos MA, Connors BW (1996) Cellular mechanisms of the augmenting response: short-term plasticity in a thalamocortical pathway. J Neurosci 16:7742-7756.

Chen Y, Bressler SL, Ding M (2006) Frequency decomposition of conditional Granger causality and application to multivariate neural field potential data. J Neurosci Methods 150:228-237.

Chen Y, Dhamala M, Schroeder CE, Ding M (2010) Current source density analysis of ongoing neural oscillations. In: Electrophysiological recording methods (Vertes RP, Stackman RW, eds), pp 27-40. Totowa, NJ: Humana.

Ching S, Cimenser A, Purdon PL, Brown EN, Kopell NJ (2010) Thalamocotical model for propofol-induced alpha rhythm associated with loss of consciousness. Proc Natl Acad Sci U S A 107:22665-22670.

Destexhe A, Sejnowski TJ (2001) Thalamocortical assemblies: how ion channels, single neurons and large-scale networks organize sleep oscillations. New York: Oxford UP.

Ding M, Bressler SL, Yang W, Liang H (2000) Short-window spectral analysis of cortical event-related potentials by adaptive multivariate autoregressive modeling: data preprocessing, model validation, and variability assessment. Biol Cybern 83:35-45.

Ding M, Chen Y, Bressler SL (2006) Granger causality: basic theory and application to neuroscience. In: Handbook of time series analysis (Winterhalder M, Schelter B, Timmer J, eds), pp 437-460. Berlin: Wiley-VCH Verlag.

Feshchenko VA, Veselis RA, Reinsel RA (2004) Propofol-induced alpha rhythm. Neuropsychobiology 50:257-266.

Flint AC, Connors BW (1996) Two types of network oscillations in neocortex mediated by distinct glutamate receptor subtypes and neuronal populations. J Neurophysiol 75:951-957.

Geweke J (1982) Measurement of linear-dependence and feedback between multiple time-series. J Am Stat Assoc 77:304-313.

Hughes SW, Crunelli V (2005) Thalamic mechanisms of EEG alpha rhythms and their pathological implications. Neuroscientist 11:357-372.

Hughes SW, Lörincz M, Cope DW, Blethyn KL, Kékesi KA, Parri HR, Juhász G, Crunelli V (2004) Synchronized oscillations at alpha and theta frequencies in the lateral geniculate nucleus. Neuron 42:253-268.

Jensen O, Gelfand J, Kounios J, Lisman JE (2002) Oscillations in the alpha band $(9-12 \mathrm{~Hz})$ increase with memory load during retention in a shortterm memory task. Cereb Cortex 12:877-882.

Jones EG (2001) The thalamic matrix and thalamocortical synchrony. Trends Neurosci 24:595-601.

Jones EG (2002) Thalamic circuitry and thalamocortical synchrony. Philos Trans R Soc Lond B Biol Sci 357:1659-1673.

Jones SR, Pinto DJ, Kaper TJ, Kopell N (2000) Alpha-frequency rhythms desynchronize over long cortical distances: a modeling study. J Comput Neurosci 9:271-291.

Jones SR, Kerr CE, Wan Q, Pritchett DL, Hämäläinen M, Moore CI (2010) Cued spatial attention drives functionally relevant modulation of the mu rhythm in primary somatosensory cortex. J Neurosci 30:13760-13765.

Kaminski M, Ding M, Truccolo WA, Bressler SL (2001) Evaluating causal 
relations in neural systems: Granger causality, directed transfer function and statistical assessment of significance. Biol Cybern 85:145-157.

Karameh FN, Dahleh MA, Brown EN, Massaquoi SG (2006) Modeling the contribution of lamina 5 neuronal and network dynamics to low frequency EEG phenomena. Biol Cybern 95:289-310.

Klimesch W, Sauseng P, Hanslmayr S (2007) EEG alpha oscillations: the inhibition-timing hypothesis. Brain Res Rev 53:63-88.

Lakatos P, Shah AS, Knuth KH, Ulbert I, Karmos G, Schroeder CE (2005) An oscillatory hierarchy controlling neuronal excitability and stimulus processing in the auditory cortex. J Neurophysiol 94:1904-1911.

Lakatos P, Karmos G, Mehta AD, Ulbert I, Schroeder CE (2008) Entrainment of neuronal oscillations as a mechanism of attentional selection. Science 320:110-113.

Lopes da Silva FH (1991) Neural mechanisms underlying brain waves: from neural mechanisms to networks. Electroencephalogr Clin Neurophysiol 79:81-93.

Lopes da Silva FH, Storm van Leeuwen W (1977) The cortical source of the alpha rhythm. Neurosci Lett 6:237-241.

Lopes da Silva FH, van Lierop TH, Schrijer CF, van Leeuwen WS (1973a) Organization of thalamic and cortical alpha rhythms: spectra and coherences. Electroencephalogr Clin Neurophysiol 35:627-639.

Lopes da Silva FH, van Lierop TH, Schrijer CF, van Leeuwen WS (1973b) Essential differences between alpha rhythms and barbiturate spindles: spectra and thalamo-cortical coherences. Electroencephalogr Clin Neurophysiol 35:641-645.

Lopes da Silva FH, Vos JE, Mooibroek J, Van Rotterdam A (1980) Relative contributions of intracortical and thalamo-cortical processes in the generation of alpha rhythms, revealed by partial coherence analysis. Electroencephalogr Clin Neurophysiol 50:449-456.

Lörincz ML, Crunelli V, Hughes SW (2008) Cellular dynamics of cholinergically induced alpha $(8-13 \mathrm{~Hz})$ rhythms in sensory thalamic nuclei in vitro. J Neurosci 28:660-671.

Maier A, Adams GK, Aura C, Leopold DA (2010) Distinct superficial and deep laminar domains of activity in the visual cortex during rest and stimulation. Front Syst Neurosci 4:pii:31.

Mehta AD, Ulbert I, Schroeder CE (2000a) Intermodal selective attention in monkeys. I: Distribution and timing of effects across visual areas. Cereb Cortex 10:343-358.

Mehta AD, Ulbert I, Schroeder CE (2000b) Intermodal selective attention in monkeys. II: Physiological mechanisms of modulation. Cereb Cortex $10: 359-370$

Mitzdorf U (1985) Current source density method and application in cat cerebral cortex: investigation of evoked potentials and EEG phenomena. Physiol Rev 65:37-100.

Mo J, Schroeder CE, Ding M (2011) Attentional modulation of alpha oscillations in macaque inferotemporal cortex. J Neurosci 31:878-882.
Niedermeyer E (2005) The normal EEG of the waking adult. In: Electroencephalography: basic principles, clinical applications and related fields (Niedermeyer E, ed), pp 149-173. Baltimore: Williams and Wilkins.

Palva S, Palva JM (2007) New vistas for $\alpha$-frequency band oscillations. Trends Neurosci 30:150-158.

Rajagovindan R, Ding M (2010) From prestimulus alpha oscillation to visual evoked response: an inverted $\mathrm{U}$ function and its attentional modulation. J Cogn Neurosci. Advance online publication. Retrieved February 28, 2011. doi:10.1162/jocn.2010.21478.

Romei V, Rihs T, Brodbeck V, Thut G (2008) Resting electroencephalogram alpha-power over posterior sites indexes baseline visual cortex excitability. Neuroreport 19:203-208.

Rougeul-Buser A, Buser P (1997) Rhythms in the alpha band in cats and their behavioural correlates. Int J Psychophysiol 26:191-203.

Schroeder CE, Tenke CE, Givre SJ, Arezzo JC, Vaughan HG Jr (1991) Striate cortical contribution to the surface-recorded pattern-reversal VEP in the alert monkey. Vision Res 31:1143-1157.

Schroeder CE, Steinschneider M, Javitt DC, Tenke CE, Givre SJ, Mehta AD, Simpson GV, Arezzo JC, Vaughan HG Jr (1995) Localization of ERP generators and identification of underlying neural processes. Electroencephalogr Clin Neurophysiol Suppl 44:55-75.

Schroeder CE, Mehta AD, Givre SJ (1998) A spatiotemporal profile of visual system activation revealed by current source density analysis in the awake macaque. Cereb Cortex 8:575-592.

Shaw JC (2003) Brain's alpha rhythm and the mind. Amsterdam: Elsevier.

Sherman SM, Guillery RW (2001) Exploring the thalamus. San Diego: Academic.

Sherman SM, Guillery RW (2002) The role of the thalamus in the flow of information to the cortex. Philos Trans R Soc Lond B Biol Sci 357:1695-1708.

Silva LR, Amitai Y, Connors BW (1991) Intrinsic oscillations of neocortex generated by layer 5 pyramidal neurons. Science 251:432-435.

Steriade M, Gloor P, Llinás RR, Lopes da Silva FH, Mesulam MM (1990) Basic mechanisms of cerebral rhythmic activities. Electroencephalogr Clin Neurophysiol 76:481-508.

Swadlow HA, Gusev AG, Bezdudnaya T (2002) Activation of a cortical column by a thalamocortical impulse. J Neurosci 22:7766-7773.

Tenke CE, Schroeder CE, Arezzo JC, Vaughan HG Jr (1993) Interpretation of high-resolution current source density profiles: a simulation of sublaminar contributions to the visual evoked potential. Exp Brain Res 94:183-192.

Worden MS, Foxe JJ, Wang N, Simpson GV (2000) Anticipatory biasing of visuospatial attention indexed by retinotopically specific alpha-band electroencephalography increases over occipital cortex. J Neurosci 20:RC63(1-6).

Zhang Y, Ding M (2010) Detection of a weak somatosensory stimulus: role of the prestimulus mu rhythm and its top-down modulation. J Cogn Neurosci 22:307-322. 